\title{
The effect of external lateral stabilization on the use of foot placement to control mediolateral stability in walking and running
}

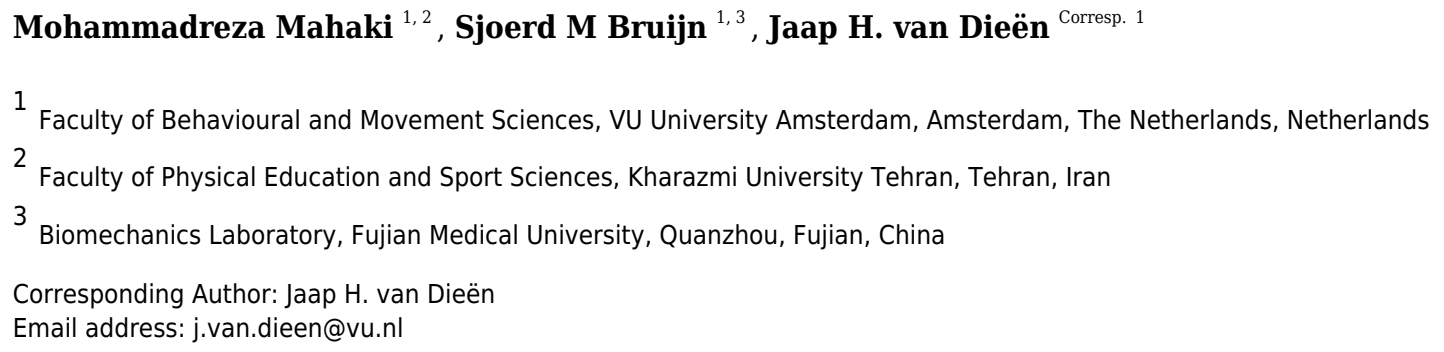

It is still unclear how humans control mediolateral (ML) stability in walking and even more so for running. Here, foot placement strategy as a main mechanism to control ML stability was compared between walking and running. Moreover, to verify the role of foot placement as a means to control ML stability in both modes of locomotion, this study investigated the effect of external lateral stabilization on foot placement control. Ten young adults participated in this study. Kinematic data of the trunk $\left(T_{6}\right)$ and feet were recorded during walking and running on a treadmill in normal and stabilized conditions. Correlation between ML trunk CoM state and subsequent ML foot placement, step width, and step width variability were assessed. Paired t-tests (either SPM1d or normal) were used to compare aforementioned parameters between normal walking and running. Twoway repeated measures ANOVAs (either SPM1d or normal) were used to test for effects of walking vs. running and of normal vs. stabilized condition. We found a stronger correlation between ML trunk CoM state and ML foot placement and significantly higher step width variability in walking than in running. The correlation between $\mathrm{ML}$ trunk CoM state and $\mathrm{ML}$ foot placement, step width, and step width variability were significantly decreased by external lateral stabilization in walking and running, and this reduction was stronger in walking than in running. We conclude that ML foot placement is coordinated to ML trunk CoM state to stabilize both walking and running and this coordination is stronger in walking than in running. 
1 The effect of external lateral stabilization on the use of foot placement

2

3

4

5

6

7

8

9

10

11 Corresponding author:

12 Jaap H. van Dieën

13 j.van.dieen@vu.nl

14

15

16

17

18

19

20

21 Wordcount: 3769
Mohammadreza Mahaki ${ }^{1,2}$, Sjoerd M. Bruijn ${ }^{1,3}$, Jaap H. van Dieën ${ }^{1}$

${ }^{1}$ Faculty of Behavioural and Movement Sciences, VU University Amsterdam, Amsterdam, The Netherlands, Netherlands

${ }^{2}$ Faculty of Physical Education and Sport Sciences, Kharazmi University Tehran, Tehran, Iran

${ }^{3}$ Biomechanics Laboratory, Fujian Medical University, Quanzhou, Fujian, China 
23 It is still unclear how humans control mediolateral (ML) stability in walking and even more so for running.

24 Here, foot placement strategy as a main mechanism to control ML stability was compared between

25 walking and running. Moreover, to verify the role of foot placement as a means to control ML stability in

26 both modes of locomotion, this study investigated the effect of external lateral stabilization on foot

27 placement control. Ten young adults participated in this study. Kinematic data of the trunk $\left(T_{6}\right)$ and feet

28 were recorded during walking and running on a treadmill in normal and stabilized conditions. Correlation

between ML trunk CoM state and subsequent ML foot placement, step width, and step width variability were assessed. Paired t-tests (either SPM1d or normal) were used to compare aforementioned parameters between normal walking and running. Two-way repeated measures ANOVAs (either SPM1d or normal) were used to test for effects of walking vs. running and of normal vs. stabilized condition. We found a stronger correlation between ML trunk CoM state and ML foot placement and significantly higher step width variability in walking than in running. The correlation between $\mathrm{ML}$ trunk CoM state and ML foot placement, step width, and step width variability were significantly decreased by external lateral stabilization in walking and running, and this reduction was stronger in walking than in running. We conclude that ML foot placement is coordinated to $\mathrm{ML}$ trunk CoM state to stabilize both walking and running and this coordination is stronger in walking than in running. 
41

42

43

44

45

46

47

48

49

\section{Introduction}

It is still unclear how humans walk and run with such ease, that is, stable and with low energy costs. Gait stability, i.e. maintaining a steady gait pattern without falling in the face of perturbations, requires control of the Center of Mass (CoM) relative to the Base of Support (BoS) [1-3]. During walking and running, motions of the CoM relative to the BoS are thought to be controlled by passive dynamics as well as active processes [1-3]. Small perturbations may be controlled by passive dynamics without Central Nervous System (CNS) involvement, and larger instabilities in the system are countered by active control, which requires sensing of perturbations, generating appropriate motor commands, and producing compensatory motions [3].

The foot placement strategy is the main mechanism to control medio-lateral (ML) stability in walking and running [4-8]. External lateral stabilization by means of a spring-like construction reduces ML CoM movement [9] and this coincided with a 24-60\% reduction in step width in walking [9-11] and 30-45\% and $12.3 \%$ reductions in step width variability in walking $[10,11]$ and running $[1]$, respectively. The coordination between CoM movements and step width is reciprocal, i.e. constraining CoM kinematics leads to adjustments of foot placement, but constraining foot placement also leads to adjustments of CoM kinematics $[12,13]$. This coordination between CoM displacement and foot placement is reflected in correlations of the CoM position and velocity during the swing phase with the subsequent foot placement [14-16]. The active nature of the control of ML stability through foot placement is supported by studies on the effects of sensory illusions induced by vibration [17], or visual perturbations [18] on this correlation, by studies that have related ML foot placement to swing phase muscle activity in control participants [19], and by studies that reported a weakened correlation in patients with neurological disorders $[20,21]$. 
Although the foot placement strategy is important for control of gait stability, to date, we do not fully understand the mechanisms underlying the control of stability of walking and even less of running. It has been shown that humans run with step widths close to zero [4]. A step width near zero may imply that there is a lower need for an accurate foot placement in running. In line with this, McClay and Cavanagh [22] demonstrated that humans run by placing the foot along the middle of the body, which aligns the vertical ground reaction forces close to the CoM, minimizes the $\mathrm{ML}$ ground reaction forces on the body from step-to-step, and minimizes the moment generated about the AP axis [23]. Thus, most of the CoM displacement is directed forward, and ML motion is relatively small [23]. Decreasing ML CoM motion may be a strategy for control of stability during running, and if this is the case, the effect of external lateral stabilization on ML displacement of CoM, step width adjustment, and correlation of preceding ML CoM state with the subsequent ML foot placement [16] will be lower in running than in walking. In the current study, we set out to test the idea that running is less dependent on foot placement to control ML stability than walking.

We hypothesized that (1) foot placement is coordinated with ML trunk CoM state in both walking and running, as reflected in a significant correlation between ML trunk CoM state during the swing phase and subsequent ML foot placement. (2) the foot placement strategy is more critical in walking than in running, as reflected in a significantly higher correlation between $M L$ trunk CoM state and subsequent $M L$ foot placement and a significantly greater step width and step width variability in walking compared to running. We further hypothesized that (3) external lateral stabilization decreases use of the foot placement strategy, as reflected by a significant reduction in the correlation between ML trunk CoM state and subsequent $\mathrm{ML}$ foot placement, alongside a significant decrease in step width, and step width variability. Since we expect more need for the foot placement strategy in walking than in running, we 
85 hypothesized that (4) the reduction in aforementioned parameters is significantly greater in walking than

86 in running ${ }^{1,2}$.

\section{2. Method}

88

89

90

91

92

93

94

95

96

97

98

99

100

101

102

103

104

\subsection{Participants}

After signing the informed consent, a convenience sample of 10 young ( 6 men, 4 women) participants (age: $27.70 \pm 4.78$ years, mass: $73.80 \pm 8.57 \mathrm{~kg}$, and height: $181.30 \pm 6.57 \mathrm{~cm}$ ) participated in this study, which had been approved by the local ethics committee of the Faculty of Behavioral and Movement Sciences of the Vrije Universiteit, Amsterdam (VCWE-2017-154). Exclusion criteria were: lower extremity injuries, history of surgery in the lower extremity, as well as any kind of impairments, medications, and infectious diseases which might affect walking mechanics or energy consumption. All of these exclusion criteria were self-reported by participants. Participants were asked to refrain from strenuous activity the day before experiments and to refrain from using coffee and alcohol on the day of the experiment.

\subsection{Experimental protocol}

Participants visited the laboratory during one session and they were measured during walking and running on a motorized treadmill in two (normal, stabilized) conditions. The participants were familiarized with walking and running on the treadmill in each condition, and they were instructed not to resist the spring forces of the stabilization frame [11]. Familiarization for each mode and each condition took about 2 minutes. Data collection started 10 minutes after the end of the familiarization protocol.

For each participant, first the conditions (normal and stabilized) were randomized and then speeds (walking at $1.25 \mathrm{~m} / \mathrm{s}$ and running at $2.08,2.50$ and $2.92 \mathrm{~m} / \mathrm{s}$ ) were randomized within each condition.

\footnotetext{
${ }^{1}$ Our initial research proposal for this project can be found at https://osf.io/mvkex/.

${ }^{2}$ The effect of running speed on aforementioned parameters, as one of our pre-planned hypotheses, can be read in the Supplementary Material file.
} 
105 Participants completed 8 trials, each trial with a duration of $5 \mathrm{~min}$. Trials were separated by a resting 106 period of approximately $5 \mathrm{~min}$.

\section{2.3. Experimental set-up}

108 A light-weight frame (mass $=1.5 \mathrm{Kg}$, see Fig 1.) was used for the external lateral stabilization condition, it was attached through a belt around the waist. Two sliders on both sides allowed participants to rotate their pelvis relative to the frame in the transverse plane, with minimal friction. Two stiff ropes attached

111 to the frame on either side, joined each other at $0.5 \mathrm{~m}$ from the frame, providing space for free arm swing.

112 From this junction, springs were attached to a slider on a vertical rail, which in turn was connected to two

113 horizontal rails placed at the height of the pelvis of the participant. Thus, the set-up did not restrict 114 movement in vertical and AP directions, nor rotations about the vertical axis, and transverse spring forces 115 acted approximately at the level of the CoM during walking and running trials (Fig 1.). Springs with spring 116 stiffness of approximately $1260 \mathrm{~N} / \mathrm{m}$ were selected in this study since in a previous study no significant 117 reductions of energy cost, step width, and step with variability were found beyond this stiffness [11].

\section{Fig 1. goes here}

\subsection{Instruments}

120

Kinematic and kinetic data during walking and running trials were obtained from an Optotrak motion analysis system (Northern Digital Inc, Ontario, Canada), sampled at 100 samples/s and from force plates

122 embedded in the treadmill (Forcelink b.v., Culemborg, the Netherlands), sampled at 1000 samples/s,

123 respectively. Clusters of three infrared markers were attached to the thorax (over the $\mathrm{T}_{6}$ spinous process)

124 and the heels.

\subsection{Data processing}

126 All our data and codes used to process the data can be found at

127 https://surfdrive.surf.nl/files/index.php/s/MzdIDXoVQwzh50z?path=\%2F. 
128 Ground reaction force data were filtered with a $10 \mathrm{~Hz}$ cut-off frequency (2nd order, bidirectional

129 Butterworth digital filter). Heel strike and toe off events were calculated from center of pressure data

130 [24]. Kinematic data from the Optotrak system were not filtered.

131 The trunk accounts for almost two-thirds of a person's body mass and the effect of its motion on control

132 of gait stability has been shown by a strong relationship between step-by- step variation in ML trunk CoM

133 state and step width during walking [14]. The mean of the three infrared markers was used to approximate

134 the ML trunk CoM position. The ML trunk CoM velocity was calculated as the first derivative of the ML

135 trunk CoM position time-series. Each step was defined from toe off to heel strike (i.e. swing phase of gait

136 cycle). Mid-stance was defined as $50 \%$ of the time between toe off and heel strike of the contralateral leg.

137 While this may not coincide with the exact moment of mid-stance, it ensures that at this moment, we are

138 absolutely certain that the foot is stationary, and thus the influence of erroneous detection of gait events

139 is minimal. The ML position of the stance foot at mid-stance was defined as the origin and ML trunk CoM,

140 and subsequent $\mathrm{ML}$ foot placement (position of the foot at the subsequent mid-stance) were expressed

141 relative to this point. To further simplify the modeling (i.e. making sure that no offset was needed), all

142 relevant variables (foot placement, ML trunk CoM, and ML trunk CoM velocity), were zero-centered by

143 subtracting the mean for each percentage of the swing phase.

144 To investigate foot placement strategy in walking, previous studies have used a regression equation which

145 predicts subsequent $\mathrm{ML}$ foot placement based on ML trunk CoM position and velocity at discrete time

146 points (e.g. mid-swing [17] or mid-stance $[14,18]$ ) of the preceding swing phase. The $\mathrm{R}^{2}$ (i.e. the ratio of

147 predicted foot placement variance to actual foot placement variance) has been reported as the primary

148 outcome in previous studies $[17,14,16] . R^{2}$ signifies the fit of regression equation which is between 0 to

$149100 \%$. The higher $\mathrm{R}^{2}$ would represent a smaller difference between predicted and actual foot placements

150 and thus would indicate a stronger correlation between ML trunk CoM state and subsequent ML foot

Peer) reviewing PDF | (2018:09:31448:3:0:NEW 20 Sep 2019) 
151 placement $[14,16] . R^{2}$ higher than $50 \%$ has been interpreted as a high correlation between $M L$ trunk CoM

152 state and subsequent ML foot placement [14]. We used the following regression equation in which ML

153 trunk CoM position and velocity time-series during swing phase predicted subsequent ML foot placement

154 [16]:

155

$$
\mathbf{F P}=\beta 1(\mathbf{i}) \cdot \operatorname{CoM}(\mathbf{i})+\beta 2(\mathbf{i}) \cdot \operatorname{VCoM}(\mathbf{i})+\varepsilon(\mathbf{i})
$$

with $\beta 1$ and $\beta 2$ being the regression coefficients, $\varepsilon$ the error, and $i$ the indicator of the $\%$ of swing phase

that was used for the prediction. Using ML trunk CoM state time-series during the preceding swing phase,

the prediction of subsequent ML foot placement was repeated for each percentage of the swing phase.

Therefore, our main outcome was the $\mathrm{R}^{2}$ time-series between predicted and actual foot placements.

Mean and variability of step width were calculated for each trial. Step width was defined as the mean of

the distances between $\mathrm{ML}$ foot placement, and step width variability was defined as the standard deviation thereof. The procedure for data processing is illustrated in Fig 2..

Fig 2. goes here

Energy costs were also measured during all conditions. Reduced energy costs in stabilized conditions would support that the control of ML stabilization requires energy consumption and differential effects between walking and running might indicate differences in these costs between these modes of locomotion. Since energy cost is not directly related to foot placement strategy, which is the main focus of this study, all the information about this parameter can be found in supplementary material.

\subsection{Statistical analysis}

170 Since our results indicated only very small differences between legs (see Supplementary Material, Fig. S1),

171 we calculated the average $R^{2}$ over legs. We selected walking at $1.25 \mathrm{~m} / \mathrm{s}$ and running at $2.5 \mathrm{~m} / \mathrm{s}$, as a 
172 representative of running speeds, to test our hypotheses ${ }^{3}$. To test whether $\mathrm{ML}$ foot placement is

173 coordinated with ML trunk CoM state in both walking at $1.25 \mathrm{~m} / \mathrm{s}$ and running at $2.50 \mathrm{~m} / \mathrm{s}$, (hypothesis

174 1), the regression coefficients $\left(\beta_{1}\right.$ and $\left.\beta_{2}\right)$ for each percentage of the swing phase in each individual

175 participant were statistically tested by one sample t-tests. Significance of one or both of these regression

176 coefficients would indicate a significant correlation between ML trunk CoM state and ML foot placement.

177 To test whether this correlation was more pronounced in walking than running, (hypothesis 2), we tested

178 for differences in $\mathrm{R}^{2}$, step width, and step width variability between normal walking at $1.25 \mathrm{~m} / \mathrm{s}$ and

179 running at $2.50 \mathrm{~m} / \mathrm{s}$, using a SPM (see below) paired t-test on the $\mathrm{R}^{2}$ time-series, and paired t-tests for

180 step width and step width variability. Subsequently, we used repeated measures ANOVA (SPM-based for

181 the $R^{2}$ time-series, normal for step width and step width variability) with Condition and Locomotion mode

182 as factors, to test for the effects of lateral stabilization (hypothesis 3 ), and we assessed the Condition $\mathrm{X}$

183 Locomotion mode interaction, to test for the differences in the effect of stabilization between walking at

$1841.25 \mathrm{~m} / \mathrm{s}$ and running at $2.50 \mathrm{~m} / \mathrm{s}$, (hypothesis 4). The SPM analysis uses random field theory to identify

185 regions in time-series that show significant effects [25]. This statistical approach captures features of the

186 entire time-series, rather than a few discrete variables. The output of SPM provides an t-value (the second

187 hypothesis) or F-value (the third and fourth hypotheses) for each sample of the $\mathrm{R}^{2}$ time-series, and a

188 threshold corresponding to $\alpha$ set at 0.05 . The values of $t$ or $F$ above the threshold indicate significant

189 effects in the corresponding portion of the time-series.

190

191

192

193

\section{Results}

The regression coefficients for $M L$ trunk CoM position $\left(\beta_{1}\right)$ were significant for all regression equations and at all instants in the swing phase, while the regression coefficients for $M L$ trunk CoM velocity $\left(\beta_{2}\right)$ were significant for most instances of the swing phase, with some exceptions. The percentage of ${ }^{3}$ Interested readers can run all analyses for each running speed by our provided codes. 
194

195

196

197

198

199

200

201

202

203

204

205

206

207

208

209

210

211

212

213

214

nonsignificant $\beta_{2}$-values was computed as the ratio of nonsignificant $\beta_{2}$-values to the total number of $\beta_{2}{ }^{-}$ values multiplied by 100 for each percentage of the swing phase (Fig 3.).Thus, since one of the coefficients was significant for all regression equations, in line with our first hypothesis, the correlation between ML trunk CoM state and subsequent ML foot placement was significant during both walking and running. The $R^{2}$ values were high, ranging between $\sim 0.52-0.85$ from $0-100 \%$ of the swing phase in walking and between 0.50-0.71 from $35-100 \%$ of the swing phase in running (Fig 4.).

In line with our second hypothesis, we found a significantly stronger correlation between ML trunk CoM state and subsequent ML foot placement in walking than in running from $0-100 \%$ of the swing phase (Fig 4. \& 5.), as well as a significantly greater step width variability $(t(1,9)=4.17, p=0.002)$ in walking than running, however the differences of step width was not significant $(t(1,9)=2.21, p=0.05)$ (Fig 6 . A and B).

\section{Fig 3., 4., and 5. go here}

In line with our third hypothesis, external lateral stabilization significantly decreased $R^{2}$ to $\sim 0.25-0.55$ and $\sim 0.36-61 \%$ during $0-100 \%$ of the swing phase in walking and running, respectively (Fig 4. and Fig 7. A). External lateral stabilization also significantly decreased step width (Condition effect; $F(1,9)=32.49, p$ $\leq 0.001$, and step width variability (Condition effect; $F(1,9)=100.24, p \leq 0.001$ )(Fig 6. A and B).

\section{Fig 6. And 7. go here}

In line with our fourth hypothesis, the effect of external lateral stabilization on $\mathrm{R}^{2}$ was larger in walking than in running (Condition X Locomotion mode effect, Fig 7. C). In addition, the effect of external lateral stabilization on step width and step width variability was larger in walking than in running (Condition X Locomotion mode effect, Fig 6 . A and B) $(F(1,9)=15.63, p=0.003$ for step width and $F(1,9)=23.21, p<$ 0.001 for step width variability). 


\section{Discussion}

216 Our results demonstrated a strong correlation between ML trunk CoM state in the swing phase of the gait

217 cycle and subsequent ML foot placement during both walking and running. ML trunk CoM state explained

218 over $50 \%$ of the variance in ML foot placement during the entire swing phase in walking and the last $65 \%$

219 of swing phase in running, respectively. Our hypothesis that the foot placement strategy is more critical

220 in walking than in running, was supported by a stronger correlation between ML trunk CoM state during

221 the swing phase and subsequent ML foot placement, as well as greater step width variability in walking

222 than in running. Furthermore, our hypothesis that external lateral stabilization significantly decreases the

223 correlation of $\mathrm{ML}$ foot placement to $\mathrm{ML}$ trunk CoM state, was also supported for both modes of

224 locomotion. This hypothesis was also supported by significant reduction in step width and step width

225 variability in the stabilized condition compared to the normal condition. The hypothesis that the foot

226 placement strategy is more critical in walking than in running was supported by stronger reductions in the

227 correlation between ML trunk CoM state and subsequent ML foot placement, and in step width, and step

228 width variability in stabilized walking than in stabilized running.

229 Our results confirmed that ML foot placement is coordinated to ML trunk CoM state in walking. Similar to previous studies, which reported that $50-84 \%$ of $M L$ foot placement variance can be explained by $\mathrm{ML}$ trunk, ML pelvis, or ML whole-body CoM state during walking [14-16], our results indicated high predictive ability of $M L$ trunk CoM state on subsequent $M L$ foot placement, with $R^{2}$ ranging between $52-85 \%$ during the entire swing phase in walking. Recently, Seethapathi and Sirinivasan [8] reported that ML foot placements relative to CoM position are predicted by mid-swing ML CoM velocity in running, with $\mathrm{R}^{2}$ values ranging $62-64 \%$. Similarly, our results indicated a high correlation between ML trunk CoM state and subsequent $M L$ foot placement $\left(R^{2}=50-71 \%\right)$ during the last $65 \%$ of the swing phase in running. The high predictive ability of ML trunk CoM state in walking and running could be due to active control of $\mathrm{ML}$ 
239

240

241

242

243

244

245

246

247

249

250

251

252

253

254

255

256

257

259

260

261

movements to movements of the upper body. Although the results of current study cannot answer the question whether active control or passive coupling is the underlying cause of this correlation, active control of ML stability through foot placement is supported by studies on the effects of sensory illusions induced by vibration [17], or visual perturbations [18] on this correlation, and by studies that have related ML foot placement to swing phase muscle activity [19]. On the other hand, we cannot rule out that the passive dynamics play a role in the correlation between $M L$ trunk CoM state and subsequent $M L$ foot placement that we report. Thus, further studies are needed to elucidate the degree to which active control contributes to foot placement coordination in walking and running.

Our results indicated that the correlation between $\mathrm{ML}$ trunk CoM state and subsequent $\mathrm{ML}$ foot placement is less strong in running than in walking. It has been suggested that the foot placement strategy begins earlier in walking when less time is available to complete the step (i.e. during walking at higher speeds) $[19,15]$. However, the more pronounced reduction in step duration in running could limit the possibility of using foot placement strategy. If this is the case, one step after a deviation of ML trunk CoM state might not be enough to restore ML stability, and more consecutive steps might be required to stabilize ML trunk CoM state in running. However, using Goal Equivalent Manifold framework, It has been reported that humans correct stride-to-stride variability both more quickly and more directly in running than in walking [26]. Such a tighter control in running might result from other stability strategies, rather than foot placement strategy. For instance, during running an absorption strategy, allowed by flexion in the lower limb, during the stance phase may be used to control the ML trunk CoM state, which may limit the need for accurate foot placement (similar as the impulse control proposed by [8]).

It has been reported that external lateral stabilization decreases ML displacement of the CoM [9], accompanied by a $24-60 \%$ reduction in step width in walking [9-11] and $30-45 \%$ in step width variability in walking $[10,11]$. Our results indicate that external lateral stabilization decreased the correlation 
262 between $M L$ trunk CoM state and subsequent ML foot placement, alongside a reduction in step width and

263 step width variability during stabilized walking. The results of the current study also indicate that external

264 lateral stabilization decreases the correlation between ML trunk CoM state and subsequent ML foot

265 placement, step width, and step width variability in running, although less so than for walking, in line with

266 a smaller decrease in step width variability of about $12 \%$ with external stabilization reported previously

267 [1]. This smaller decrease may suggest that subjects need more foot placement strategy during stabilized

268 running than during stabilized walking. This would appear to contradict the notion that the foot placement

269 strategy is less important during normal running than normal walking. However, there may be several

270 alternative explanations. First of all, the external lateral stabilization may have different effects on ML

271 stability in running and walking; it may be less effective during running, as the ML forces may affect body

272 movements differently during the flight phase in running compared to the single leg stance phase in

273 walking. In single leg stance, the spring forces and ground reaction forces on the stance leg may produce

274 a rotational couple, which does not occur during the flight phase in running. It could be that this rotational

275

276

277

278

279

280

281

282

283

284

component is key to stabilizing subjects. Thus, the stabilizing effect may be different between walking and

running, but for now, this remains speculation. A second explanation, may be that subjects do not experience the frame as sufficiently stabilizing in running and thus do not "offload" control to the frame as much as they do in walking. However, participants were familiarized with all conditions, and did not express feelings of discomfort during any of the conditions, rendering this unlikely.

\section{Conclusion}

$\mathrm{ML}$ trunk CoM state explained over $50 \%$ of the variance in $\mathrm{ML}$ foot placement during the entire swing phase in walking, and the last $65 \%$ of swing phase in running. This suggests that $M L$ foot placement is correlated to ML trunk CoM state to actively control ML stability at the end of gait cycle in walking and running. Still the passive dynamics coupling between $\mathrm{ML}$ trunk CoM movement and ML foot placement might play role on this correlation. The foot placement strategy appears more critical in walking than in

Peer] reviewing PDF | (2018:09:31448:3:0:NEW 20 Sep 2019) 
286

287

288

289

290

291

292

293

294

295

296

297

298

299

300

301

302

303

304

305

306

307

308

309

310

311

312

313

314

315

316

317

318

319

320

running, as the correlation between $\mathrm{ML}$ trunk CoM state and subsequent $\mathrm{ML}$ foot placement was higher

in walking than running. External lateral stabilization decreased this correlation, step width, and step width variability in both walking and running, with stronger reductions during the former. This may imply

that there is a higher need for an accurately coordinated foot placement in walking.

\section{Acknowledgements}

We like to thank Leon Schutte and Hans de Koning for experimental help.

\section{References}

1. Arellano, C.J. and R. Kram, The energetic cost of maintaining lateral balance during human running. Journal of Applied Physiology, 2011. 112(3): p. 427-434.

2. Bauby, C.E. and A.D. Kuo, Active control of lateral balance in human walking. Journal of biomechanics, 2000. 33(11): p. 1433-1440.

3. Kuo, A.D. and J.M. Donelan, Dynamic principles of gait and their clinical implications. Physical therapy, 2010. 90(2): p. 157.

4. Arellano, C.J. and R. Kram, The effects of step width and arm swing on energetic cost and lateral balance during running. Journal of biomechanics, 2011. 44(7): p. 1291-1295.

5. Bruijn, S.M. and J.H. van Dieën, Control of human gait stability through foot placement. Journal of The Royal Society Interface, 2018. 15(143): p. 20170816.

6. Donelan, J.M. and R. Kram, Mechanical and metabolic determinants of the preferred step width in human walking. Proceedings of the Royal Society of London B: Biological Sciences, 2001. 268(1480): p. 1985-1992.

7. Reimann, H., T. Fettrow, and J.J. Jeka, Strategies for the control of balance during locomotion. Kinesiology Review, 2018. 7(1): p. 18-25.

8. Seethapathi, N. and M. Srinivasan, Step-to-step variations in human running reveal how humans run without falling. eLife, 2019. 8: p. e38371.

9. Dean, J.C., N.B. Alexander, and A.D. Kuo, The effect of lateral stabilization on walking in young and old adults. IEEE Transactions on Biomedical Engineering, 2007. 54(11): p. 1919-1926.

10. Donelan, J.M., D.W. Shipman, R. Kram, and A.D. Kuo, Mechanical and metabolic requirements for active lateral stabilization in human walking. Journal of biomechanics, 2004. 37(6): p. 827-835.

11. ljmker, T., H. Houdijk, C.J. Lamoth, P.J. Beek, and L.H. van der Woude, Energy cost of balance control during walking decreases with external stabilizer stiffness independent of walking speed. Journal of biomechanics, 2013. 46(13): p. 2109-2114.

12. Arvin, M., M. Mazaheri, M.J. Hoozemans, M. Pijnappels, B.J. Burger, S.M. Verschueren, and J.H. van Dieën, Effects of narrow base gait on mediolateral balance control in young and older adults. Journal of biomechanics, 2016. 49(7): p. 1264-1267. 
321

322

323

324

325

326

327

328

329

330

331

332

333

334

335

336

337

338

339

340

341

342

343

344

345

346

347

348

349

350

351

352

353

354

355

356

357

358

359

360

13. Arvin, M., J.H. van Dieën, and S.M. Bruijn, Effects of constrained trunk movement on frontal plane gait kinematics. Journal of biomechanics, 2016. 49(13): p. 3085-3089.

14. Hurt, C.P., N. Rosenblatt, J.R. Crenshaw, and M.D. Grabiner, Variation in trunk kinematics influences variation in step width during treadmill walking by older and younger adults. Gait \& posture, 2010. 31(4): p. 461-464.

15. Stimpson, K.H., L.N. Heitkamp, J.S. Horne, and J.C. Dean, Effects of walking speed on the step-bystep control of step width. Journal of biomechanics, 2018. 68: p. 78-83.

16. Wang, Y. and M. Srinivasan, Stepping in the direction of the fall: the next foot placement can be predicted from current upper body state in steady-state walking. Biology letters, 2014. 10(9): p. 20140405.

17. Arvin, M., M. Hoozemans, M. Pijnappels, J. Duysens, S.M.P. Verschueren, and J. Van Dieen, Where to step? Contributions of stance leg muscle spindle afference to planning of mediolateral foot placement for balance control in young and older adults. Frontiers in Physiology, 2018. 9: p. 1134.

18. Reimann, H., T. Fettrow, E.D. Thompson, and J.J. Jeka, Neural control of balance during walking. Frontiers in Physiology, 2018. 9.

19. Rankin, B.L., S.K. Buffo, and J.C. Dean, A neuromechanical strategy for mediolateral foot placement in walking humans. Journal of neurophysiology, 2014. 112(2): p. 374-383.

20. Dean, J.C. and S.A. Kautz, Foot placement control and gait instability among people with stroke. Journal of rehabilitation research and development, 2015. 52(5): p. 577.

21. Stimpson, K.H., L.N. Heitkamp, A.E. Embry, and J.C. Dean, Post-stroke deficits in the step-by-step control of paretic step width. Gait \& posture, 2019. 70: p. 136-140.

22. McClay, I. and P. Cavanagh, Relationship between foot placement and mediolateral ground reaction forces during running. Clinical Biomechanics, 1994. 9(2): p. 117-123.

23. Cavanagh, P.R., The biomechanics of lower extremity action in distance running. Foot \& ankle, 1987. 7(4): p. 197-217.

24. Roerdink, M., C.J. Lamoth, and P.J. Beek, Online gait event detection using a large force platform embedded in a treadmill. Journal of biomechanics, 2008. 41(12): p. 2628-2632.

25. Pataky, T.C., M.A. Robinson, and J. Vanrenterghem, Vector field statistical analysis of kinematic and force trajectories. Journal of biomechanics, 2013. 46(14): p. 2394-2401.

26. Dingwell, J.B., N.K. Bohnsack-McLagan, and J.P. Cusumano, Humans Control Stride-to-Stride Stepping Movements Differently for Walking and Running, Independent of Speed. Journal of biomechanics, 2018. 76: p. 144-151.

\section{Figure captions}

Fig 1. (A) Schematic representation of the experimental set up. Inset (B) shows the stabilization in more detail. (1) frame; (2) springs; (3) height-adjustable horizontal rail; (4) ball-bearing trolley freely moving in anterior-posterior direction; (5) slider freely moving in vertical direction; (6) vertical rail; and (7) rope attached to frame.

Fig 2. Flow of data processing adopted in this study.

Peer) reviewing PDF | (2018:09:31448:3:0:NEW 20 Sep 2019) 
362 Fig 3. The $\%$ of nonsignificant $\beta_{2}$ 's during normal and stabilized conditions in walking and running trials 363 per each \% of swing phase.

Fig 4. The ability of $M L$ trunk CoM state to predict subsequent $M L$ foot placement $\left(R^{2}\right)$ during normal 366 (solid) and stabilized (dashed) conditions in walking (blue) and running (green). The shaded regions indicate standard error of $\mathrm{R}^{2}$.

Fig 5. The differences of $\mathrm{R}^{2}$ between normal walking and running. The shaded areas indicate significant effects in the corresponding portion of the swing phase (based on the results of SPM paired t-test).

Fig 6. Condition effect: The effect of external lateral stabilization on (A) step width and (B) step width variability in walking and running. \# represents the significant differences of step width and step width variability between normal and stabilized conditions (based on the results of Bonferroni post-hoc tests). and running (based on the results of paired t-test). The error bars represent the standard deviation.

Fig 7. (A) Condition effect: The effect of external lateral stabilization on $R^{2}$ in walking and running. (B) Locomotion mode effect: The differences of $\mathrm{R}^{2}$ between walking and running in both conditions (normal \& stabilized). (C) Interaction effect (condition $\times$ locomotion mode effect): The differences of external lateral stabilization effect on $\mathrm{R}^{2}$ between walking and running. The shaded areas indicate significant effects in the corresponding portion of the swing phase. 


\section{Figure 1}

Schematic representation of the experimental set up

(A) Schematic representation of the experimental set up. Inset (B) shows the stabilization in more detail. (1) frame; (2) springs; (3) height-adjustable horizontal rail; (4) ball-bearing trolley freely moving in anterior-posterior direction; (5) slider freely moving in vertical direction; (6) vertical rail; and (7) rope attached to frame. 


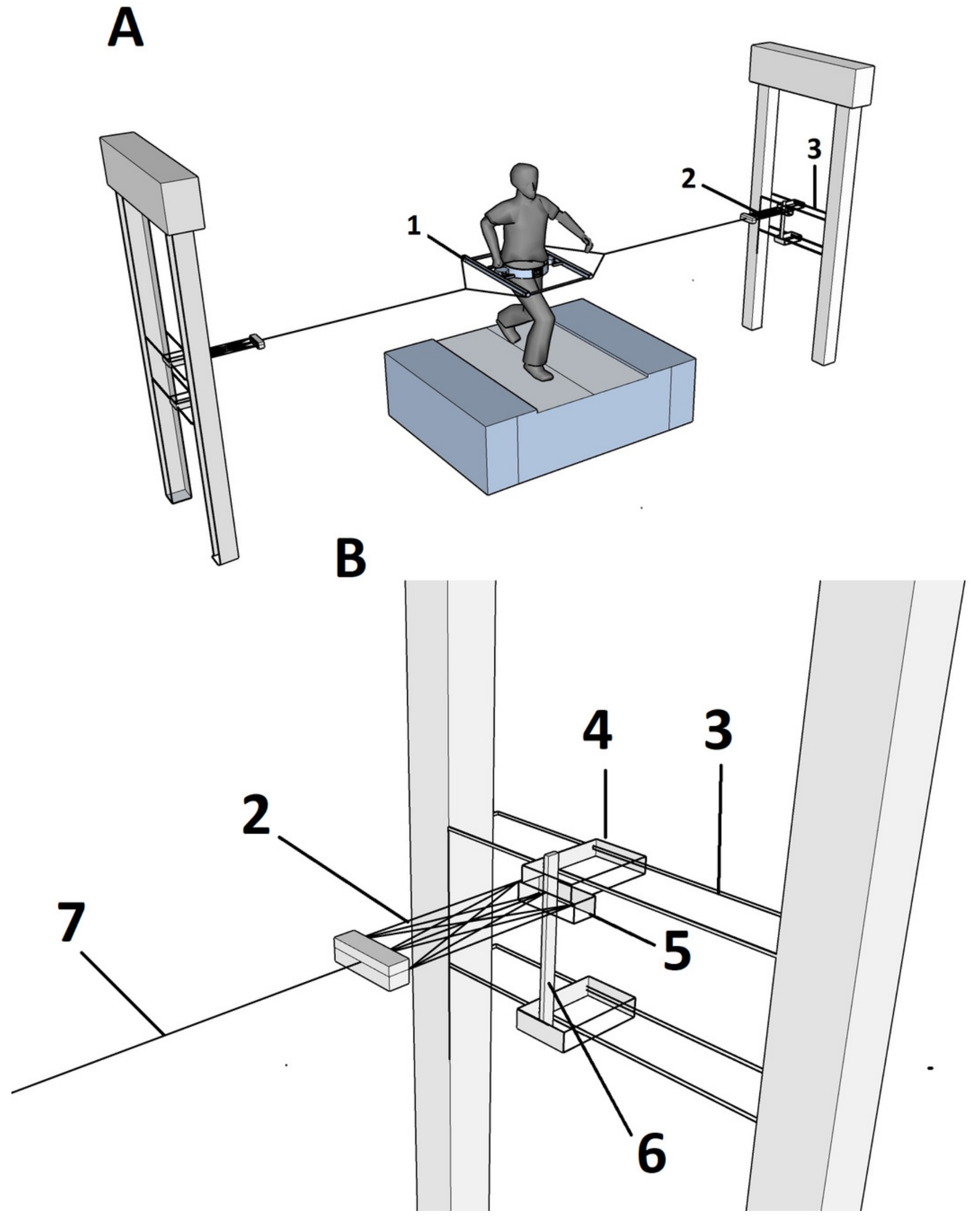


Figure 2

Flow of data processing adopted in this study. 


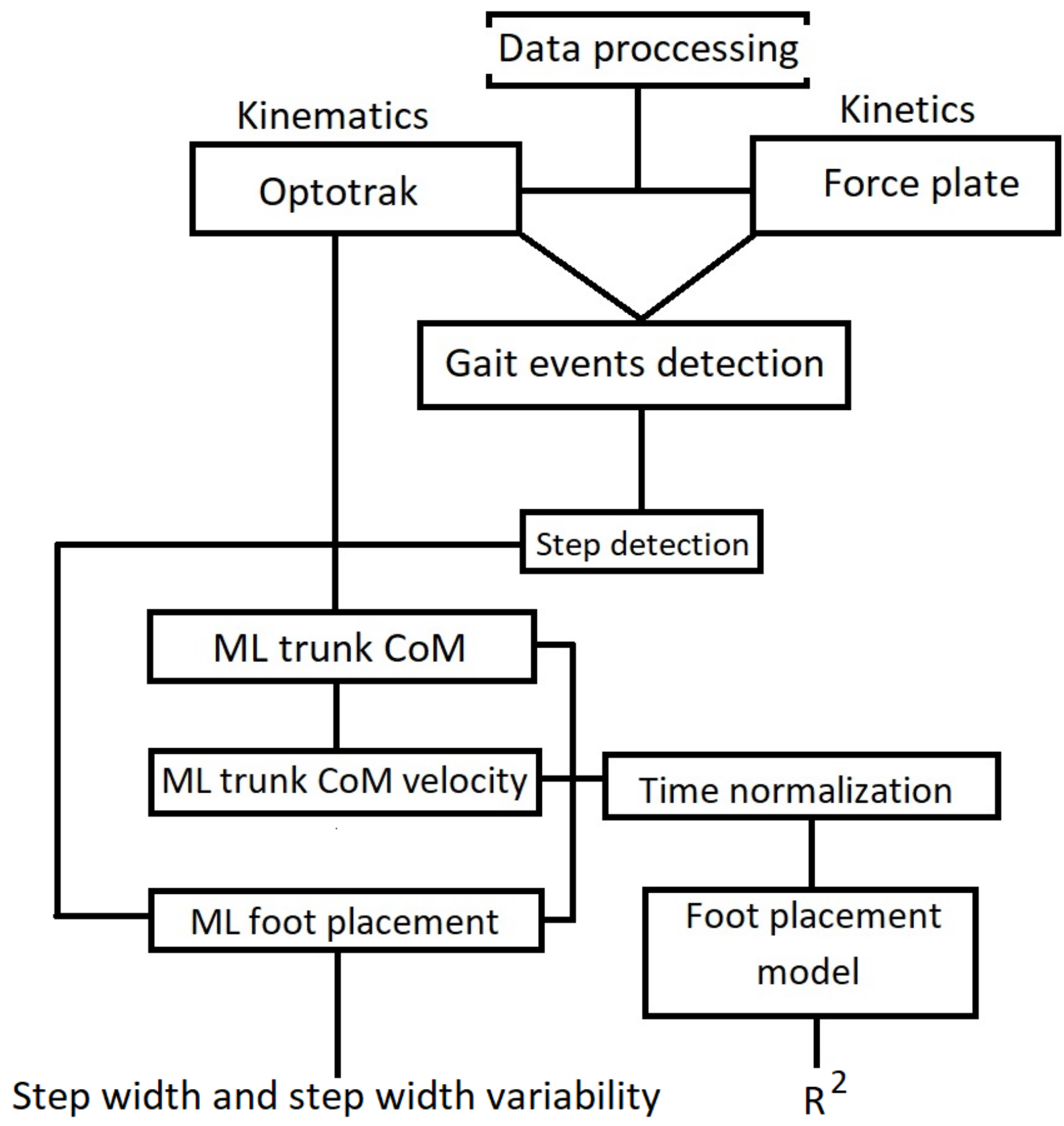


Figure 3

The $\%$ of nonsignificant $\beta_{2}$ 's during normal and stabilized conditions in walking and running trials per each \% of swing phase.

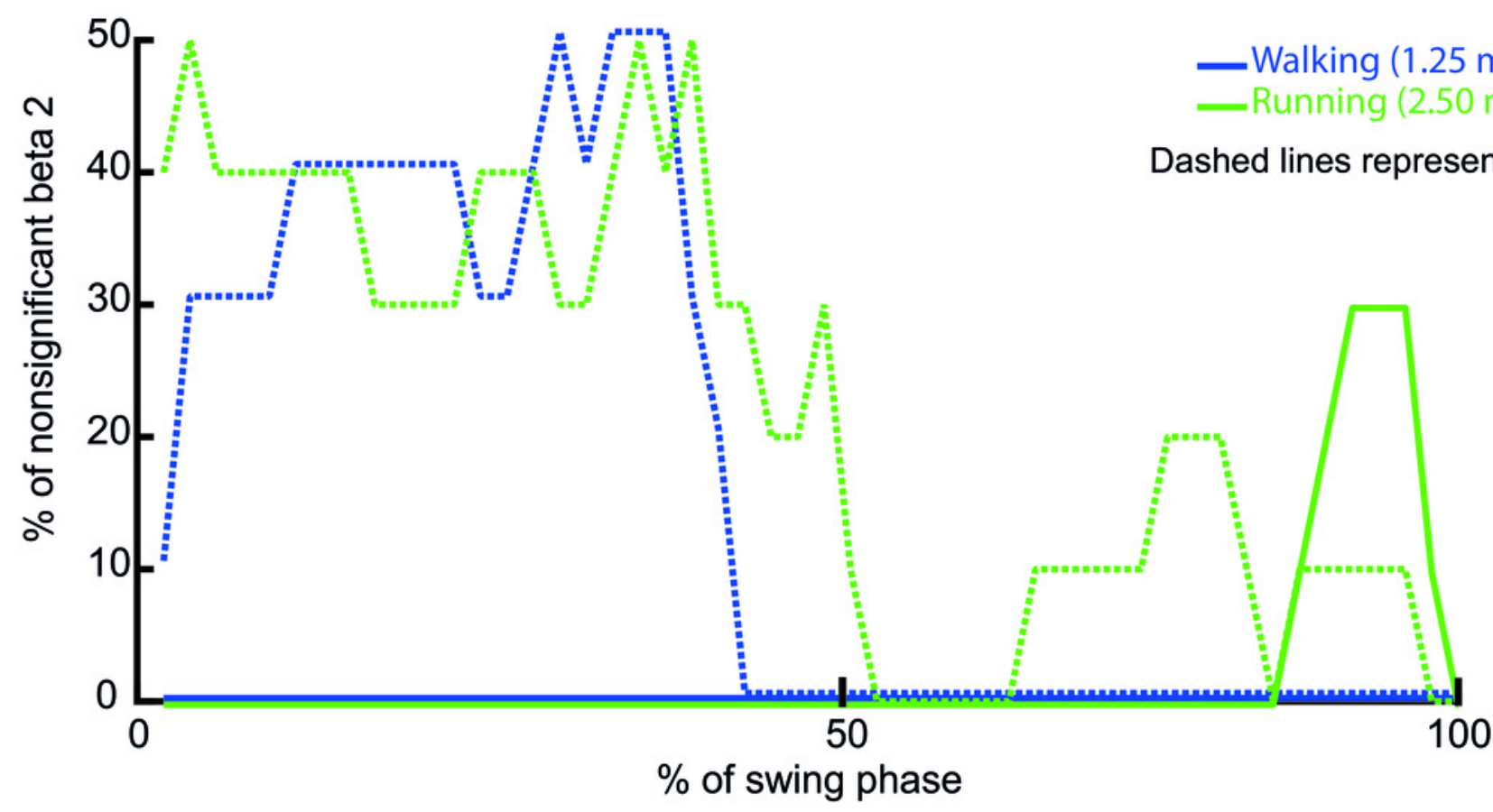


Figure 4

The ability of ML trunk CoM state to predict subsequent ML foot placement $\left(R^{2}\right)$ during normal (solid) and stabilized (dashed) conditions in walking (blue) and running (green). The shaded regions indicate standard error of $R^{2}$.

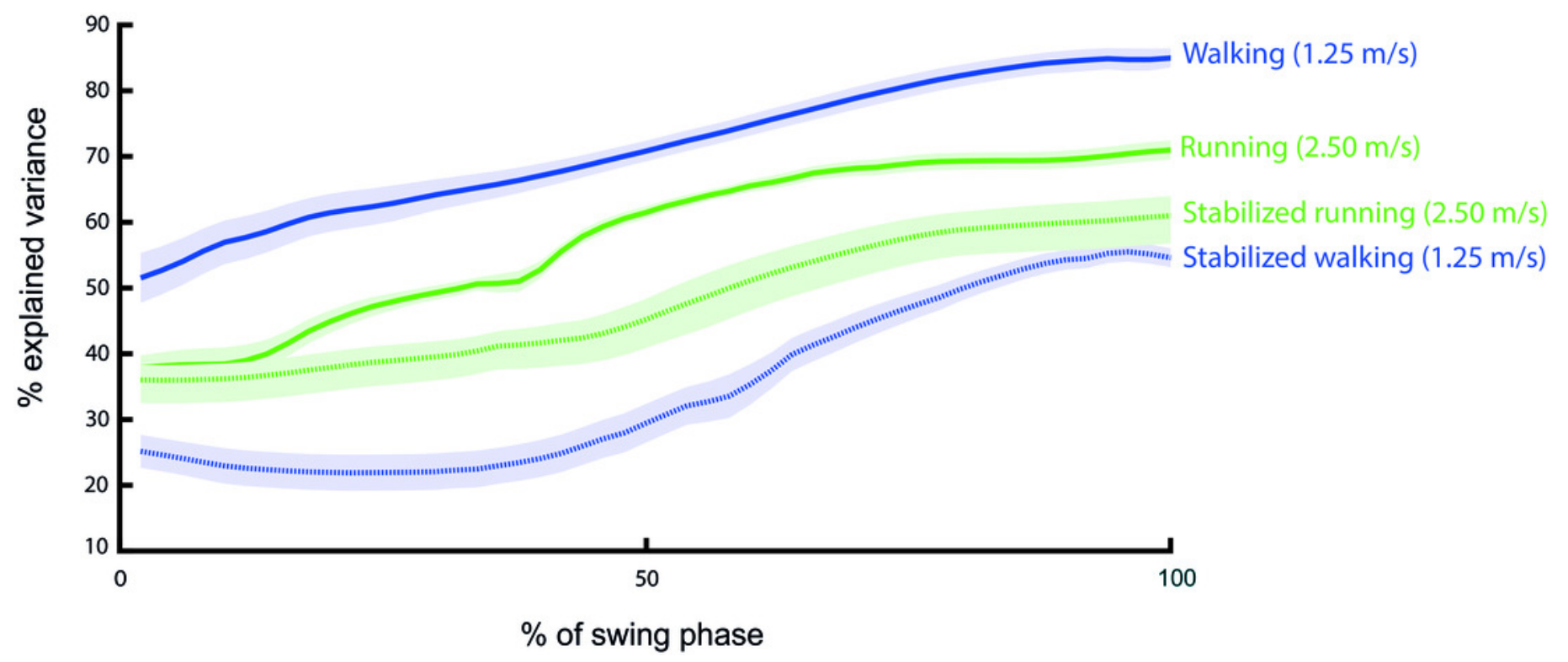


Figure 5

The differences of $R^{2}$ between normal walking and running. The shaded areas indicate significant effects in the corresponding portion of the swing phase (based on the results of SPM paired t-test).

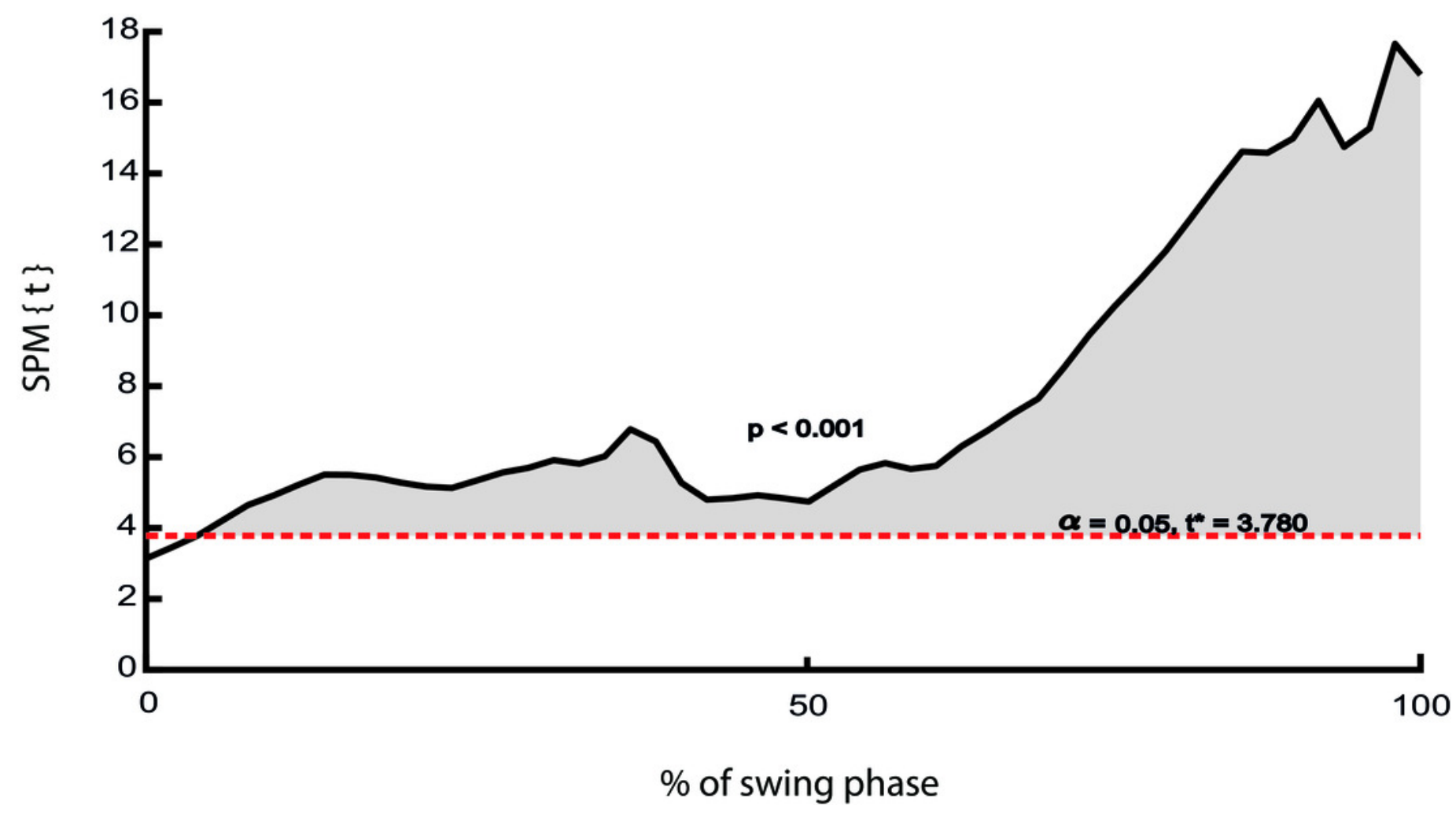




\section{Figure 6}

The effect of external lateral stabilization on (A) step width and (B) step width variability.

Condition effect: The effect of external lateral stabilization on (A) step width and (B) step width variability in walking and running. \# represents the significant differences of step width and step width variability between normal and stabilized conditions (based on the results of Bonferroni post-hoc tests). * represents the significant differences of step width and step width variability between normal walking and running (based on the results of paired t-test). The error bars represent the standard deviation.

A

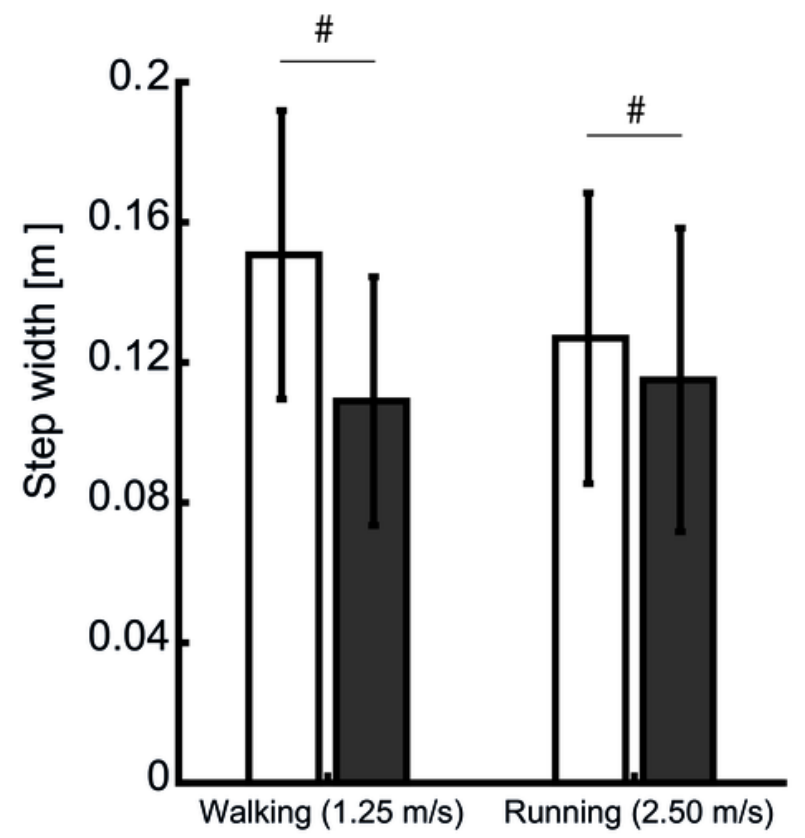

B

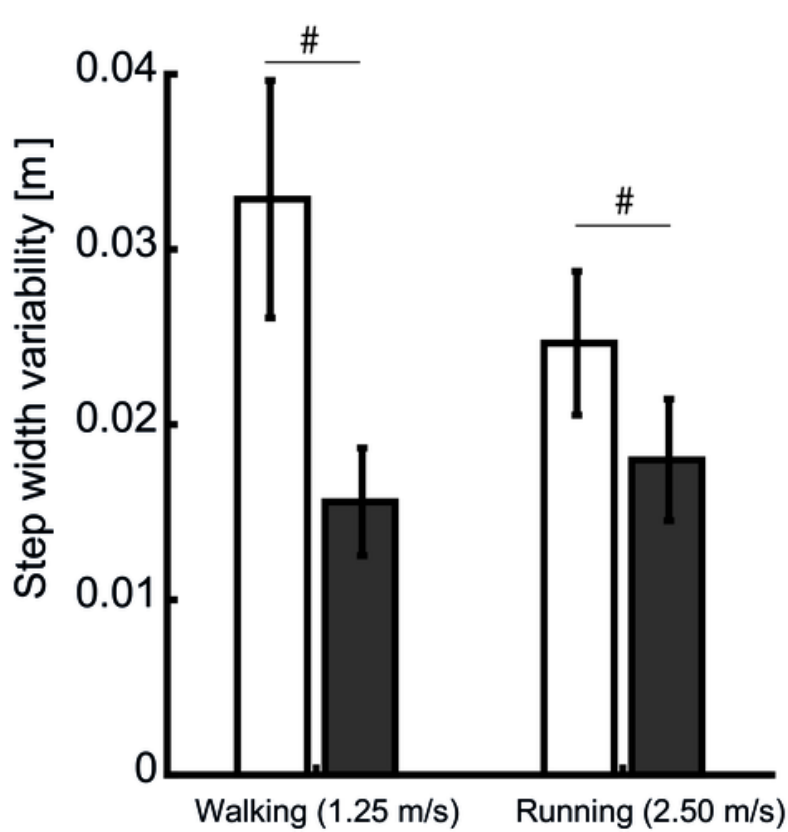


Figure 7

The effect of lateral stabilization on $\mathrm{R}^{2}$ in walking and running

(A) Condition effect: The effect of external lateral stabilization on $\mathrm{R}^{2}$ in walking and running.

(B) Locomotion mode effect: The differences of $\mathrm{R}^{2}$ between walking and running in both conditions (normal \& stabilized). (C) Interaction effect (condition $\times$ locomotion mode effect):

The differences of external lateral stabilization effect on $\mathrm{R}^{2}$ between walking and running. The shaded areas indicate significant effects in the corresponding portion of the swing phase.

A

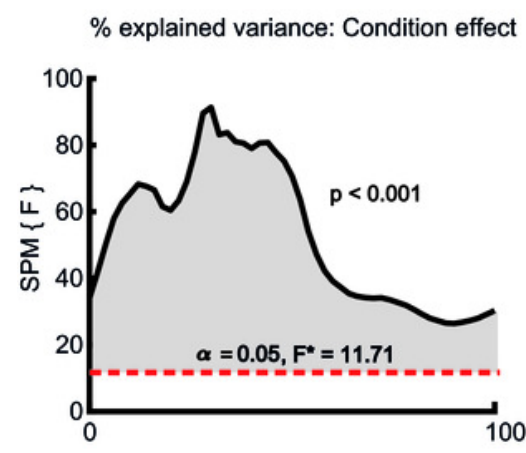

B

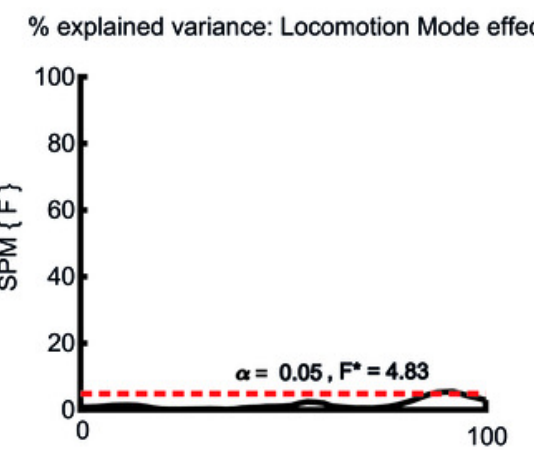

C

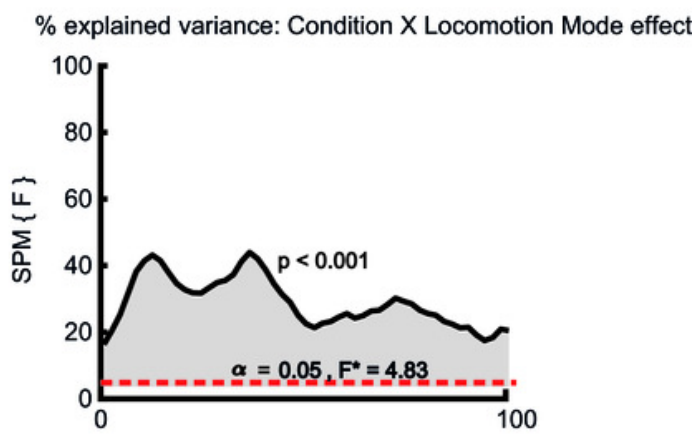

